October 23, 2018 13:9 WSPC/Guidelines bhthird1

\title{
Black Holes and the Third Law of Thermodynamics
}

\author{
F. Belgiorno* \\ Dipartimento di Fisica, Università degli Studi di Milano, \\ Via Celoria 16, 20133 Milano, Italy, and \\ I.N.F.N., sezione di Milano, Italy \\ M. Martellini ${ }^{\dagger}$ \\ Dipartimento di Fisica, Università degli Studi dell'Insubria, \\ Via Valleggio 11, Como, and \\ I.N.F.N., sezione di Milano, Italy, and \\ Landau Network at "Centro Volta", Como, Italy
}

\begin{abstract}
We discuss in the framework of black hole thermodynamics some aspects relative to the third law in the case of black holes of the Kerr-Newman family. In the light of the standard proof of the equivalence between the unattainability of the zero temperature and the entropic version of the third law it is remarked that the unattainability has a special character in black hole thermodynamics. Also the zero temperature limit which obtained in the case of very massive black holes is discussed and it is shown that a violation of the entropic version in the charged case occurs. The violation of the Bekenstein-Hawking law in favour of zero entropy $S_{E}=0$ in the case of extremal black holes is suggested as a natural solution for a possible violation of the second law of thermodynamics. Thermostatic arguments in support of the unattainability are explored, and $S_{E}=0$ for extremal black holes is shown to be again a viable solution. The third law of black hole dynamics by W.Israel is then interpreted as a further strong corroboration to the picture of a discontinuity between extremal states and non-extremal ones.
\end{abstract}

PACS: 04.70.Dy, 05.70.-a

\section{Introduction}

The third law of thermodynamics is explored in light of the correspondences existing between standard thermodynamics and black hole thermodynamics 123145 . We summarize some aspects of the third law in standard thermodynamics and also some related results in black hole thermodynamics. In black hole thermodynamics, the unattainability (U) of $T=0$ holds and the entropic version (N) fails; it is underlined that $(\mathrm{U})$ has a special status, is clearly not equivalent to $(\mathrm{U})$ as it is realized in standard thermodynamics. Moreover, the limit $T \rightarrow 0^{+}$obtained for black hole

*E-mail address: belgiorno@mi.infn.it

${ }^{\dagger}$ E-mail address: maurizio.martellini@uninsubria.it 
mass $M \rightarrow \infty$ is explored. (N) is shown to hold in the case of uncharged black holes; at the same time, (U) holds also for the charged case. Then we focus our attention on extremal states. An analysis in a thermostatic framework suggests that it is appealing to abandon the Bekenstein-Hawking law in the case of the extremal black holes and to assume that extremal black hole have zero entropy $S_{E}=0$. Thermostatic reasons for the unattainability (U) of extremal states are then analyzed; in particular, if $S_{E}=0$, then it is possible to corroborate the unattainability also in a thermostatic framework. Unattainability would mean simply the impossibility of a process violating the second law of thermodynamics. The analysis of the implications of Israel's proof 4 , to be interpreted in a irreversible thermodynamic framework, corroborates the statement that extremal states have to be discontinuous with respect to the equilibrium thermodynamic space of non-extremal ones.

The plan of the paper is the following. In sect. 2 a review of discussions and results about third law of thermodynamics in black hole physics 1231456 and of results about extremal black hole entropy 78910 , a short review of the status of the third law in standard thermodynamics 1112131415 and Nernst's theorem is made in sect. 3. In sect. 4 an analysis of the limit $T \rightarrow 0^{+}$in black hole thermodynamics follows. A first analysis concerns extremal states and then the limit $T \rightarrow 0^{+}$associated with infinitely massive black holes is explored.

In sect. 5 the thermodynamic properties of extremal black holes are enhanced. In sect. 6] Carathéodory's approach to black hole thermodynamics is applied in order to study the properties of the extremal surface $T=0$ and it is shown that, by including it in the thermodynamic manifold, one cannot obtain a well-behaved thermodynamic foliation. $S_{E}=0$ is proposed as a solution to this problem and the introduction of this discontinuity between non-extremal states and extremal ones is shown to be viable. In sect. 7 unattainability is discussed in a thermostatic framework. In sect. 8 a translation of Israel's result 4 in a irreversible thermodynamic frame is made. Appendices A,B,C and D concern further aspects of the physics involved in our paper.

\section{The third law}

In standard thermodynamics there are two formulations of the third law. The entropic version of Nernst's theorem (N) states that, for every system, if one considers the entropy as a function of the temperature $T$ and of other macroscopic parameters $x^{1}, \ldots, x^{n}$, the entropy difference $\Delta_{T} S \equiv S\left(T, x^{1}, \ldots, x^{n}\right)-S\left(T, \bar{x}^{1}, \ldots, \bar{x}^{n}\right)$ goes to zero as $T \rightarrow 0^{+}$

$$
\lim _{T \rightarrow 0^{+}} \Delta_{T} S=0
$$

for any choice of $\left(x^{1}, \ldots, x^{n}\right)$ and of $\left(\bar{x}^{1}, \ldots, \bar{x}^{n}\right)$. Thus $(\mathrm{N})$ requires that the limit $\lim _{T \rightarrow 0^{+}} S\left(T, x^{1}, \ldots, x^{n}\right)$ is a constant $S_{0}$ which does not depend on the macroscopic parameters $x^{1}, \ldots, x^{n}$. Planck's restatement of Nernst's postulate fixes the 
entropy constant $S_{0}$ at $T=0$ to be zero ${ }^{\text {a }}$. Sometimes, the $(\mathrm{N})$ version is expressed by saying that the zero temperature states of a system are isentropic. The latter statement is at least ambiguous, if the entropy is allowed to be discontinuous; the statement involving the limit of $S$ as $T \rightarrow 0^{+}$is in any case to be preferred. The unattainability version (U) can be expressed as the impossibility to reach the absolute zero of the temperature by means of a finite number of thermodynamic processes. Both the above formulations are due to Nernst. It is generally assumed that the two formulations are equivalent. Actually, this equivalence is not automatic, as it results from a discussion in 131415. We mean to come back to this topic in sect. [5] where its relevance in black hole thermodynamics is enhanced.

The third law of thermodynamics in black hole physics has been discussed since the formulation of the laws of black hole mechanics 1. In fact, in 1 the analogy between the standard third law, in the form of unattainability (U) of the absolute zero temperature, and the unattainability of the extremal states by means of a finite number of physical processes is remarked 2]. A more recent result about the unattainability is found in 4 , where the unattainability is rigorously obtained under suitable hypotheses. We discuss in the following this result further on. (N) is explored in the framework of black hole thermodynamics e.g. in 3 . Therein it is stressed the failure of the entropic side of the third law in black hole thermodynamics. On the side of $(\mathrm{N})$, we recall also some results obtained in the framework of gravitational partition function calculations. In 78 the entropy of an extremal Reissner-Nordström black hole is predicted to be zero and this result is related with the boundary structure of the spacetime. Analogous statements are found in 9 and a further corroboration of $S=0$ for extremal Reissner-Nordström black holes appears in 10, where a semiclassical calculation of the entropy in canonical quantum gravity is made. The latter approach leads to a result that introduces a violation of the Bekenstein-Hawking law and is in agreement with the requirement of isentropic zero temperature states. This isentropy is not equivalent to $(\mathrm{N})$, even if it seems to match Planck's requirement of zero entropy for any system at the absolute zero of the temperature. A deeper discussion is found in the following sections. On the other hand, superstring theory and supergravity allow again the opposite result in which $S=A / 4$ for extremal black holes. We don't discuss herein the latter approach.

Doubts about the validity of thermodynamics for values of $T$ very near the absolute zero have been raised, when finite-size systems are taken into account. A thermodynamic description of a "standard" system below a given temperature is impossible according to Planck, because of a reduction of the effective degrees of freedom making impossible even to define an entropy. Only statistical mechanics

a This is mandatory in the case of homogeneous thermodynamics, as is shown in 19 and it is trivial to prove. 
is then viable. In 16 this breakdown of thermodynamics near the absolute zero is shown to occur because of finite size effects, which make impossible to neglect statistical fluctuations in the calculation of thermodynamic quantities like e.g. T, S. On the black hole side of this topics, arguments that are in some sense of the same nature as Planck's ones are found in 17. In fact, therein hints against the possibility of a thermal description of near extremal states, because of the occurrence of uncontrollable thermodynamic fluctuations, are given, and are related to the finite size nature of black holes (note also that a notion of thermodynamic limit is missing in the black hole case).

We also limit ourselves to point out that an unconventional discussion of black hole thermodynamics in a non-equilibrium framework and a unconventional discussion also of the third law are found in 18 .

Herein, we discuss the extremal limit of black hole thermodynamics, and also the third principle in black hole thermodynamics with respect to the limit $T \rightarrow 0^{+}$ obtained for infinite black hole mass $M \rightarrow \infty$.

\section{The equivalence $(\mathrm{U}) \Leftrightarrow(\mathrm{N})$ revisited. Landsberg's analysis}

In the following, we first focus our attention on the relation between $(\mathrm{N})$ and $(\mathrm{U})$ and on the possibility to de-link the unattainability from the entropic version of Nernst's theorem. The double implication $(\mathrm{U}) \Leftrightarrow(\mathrm{N})$, according to the analysis developed by Landsberg in 1315, relies on some hypotheses that it is interesting to recall. In the following sections we discuss some properties of black hole thermodynamics near the absolute zero in the light of this analysis. For a detailed discussion about the third law in standard thermodynamics see also 1920.

\section{1. $(U) \Rightarrow(N)$}

A detailed analysis shows that in standard thermodynamics unattainability (U) implies (N) if the following conditions a), b), c) are satisfied 1315 .

a) The stability condition $(\partial S / \partial T)_{x}>0$ is satisfied for any transformation such that the external parameters (or deformation coordinates; in our discussion we include constitutive coordinates in the set of deformation coordinates), collectively indicated by means of $x$, are kept fixed; these transformations are called isometric transformations 21. As a consequence, the heat capacity $C_{x}$ at constant deformation parameters $x$ has to be positive $\forall T>0$.

This hypothesis is in general ensured by the convexity/concavity properties of the thermodynamic potentials; as a consequence, in Landsberg's works 1314115] this hypothesis is actually assumed to be always satisfied, so it is not considered as a possible cause of failure of the double implication $(\mathrm{U}) \Leftrightarrow(\mathrm{N})$. Given also the peculiar thermodynamic properties of black holes, we must choose a) as a further hypothesis 
to be discussed.

b) There are no multiple branches in thermodynamic configuration space.

This condition is introduced in order to avoid some pathological situations discussed in 1314 (no physical behavior corresponds to them; see fig. 11).

c) There is no discontinuity in thermodynamic properties of the system near the absolute zero.

In 13 a careful discussion of the conditions to be satisfied in order to ensure (U) is contained. In particular, by following 13 , if a),b),c) hold and moreover (N) fails, then $T=0$ is attainable. If a),b) and c) hold, then (U) implies (N). If a),b) hold and $(\mathrm{N})$ fails, then $(\mathrm{U})$ implies that a discontinuity near the absolute zero has to occur, and such a discontinuity has to prevent the attainability of $T=0$ (violation of c)) 13 . Anyway, in standard thermodynamics a violation of c) is ruled out 13 , and (U) is associated with the impossibility to get states at $T>0$ isentropic to states at $T=0$.

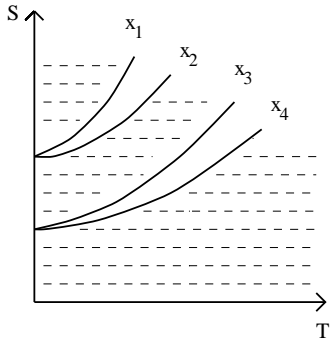

(a)

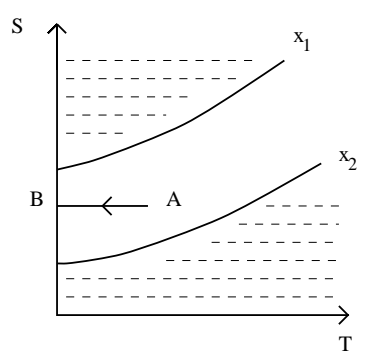

Fig. 1. (a) Multi-branches structure of the thermodynamic space. According to Landsberg, it implies the validity of $(\mathrm{U})$ and the violation of $(\mathrm{N})$. (b) Violation of $(\mathrm{N})$ that implies a violation of $(\mathrm{U})$, due to the presence of the isentropic AB. Landsberg conjectures that (U) holds if a discontinuity near $T=0$ occurs. See also the text. In (a) and (b) the dashed regions are forbidden.

A further condition "entropies don't diverge as $T \rightarrow 0^{+}$" is also introduced in 1315 in order to take into account the actual behavior near zero temperature of the standard thermodynamic systems. In fact, a priori, (N) could hold also if one should find a divergence in the entropy as $T \rightarrow 0^{+}$not depending on $x$ 13. However, we don't introduce this further hypothesis in view of our analysis in sect. 4.2

About possible failures of the implication $(\mathrm{U}) \Rightarrow(\mathrm{N})$ see also 22 .

As far as black hole thermodynamics is concerned, we note that the violation of $(\mathrm{N})$ is such that $(\mathrm{U})$ cannot be interpreted as absence of isentropic transformations which allow to reach $T=0$. See sect. [6 
3.2. $(N) \Rightarrow(U)$

A full implication $(\mathrm{N}) \Rightarrow(\mathrm{U})$ is possible in the case of thermodynamic processes which consist of an alternate sequence of quasi-static adiabatic transformations and quasi-static isothermal transformations (class $\mathrm{P}(\mathrm{x})$ according to 1314 $)$. Actually, a more general notion of unattainability can be assumed, that is, "zero temperature states don't occur in the specification of attainable states of systems". This is almost literally the (U4) principle introduced by Landsberg 1314. (U4)-unattainability states that no process allows to reach states at $T=0$, even as transient nonequilibrium states. Then $(\mathrm{N})$ can fail and $(\mathrm{U})$ can still be valid. In general, the latter hypothesis allows a de-linking of $(\mathrm{U})$ and $(\mathrm{N})$ and implies that $(\mathrm{N}) \not(\mathrm{U})$ and $(\mathrm{U}) \nRightarrow(\mathrm{N})$ 1314. But such a de-linking occurs under particular conditions. The failure of the implication $(\mathrm{U}) \Rightarrow(\mathrm{N})$ requires again a rejection of one of the hypotheses b),c) above, whereas $(\mathrm{N}) \Rightarrow(\mathrm{U})$ fails if processes not belonging to the aforementioned class $\mathrm{P}(\mathrm{x})$ allow to reach $T=01314$.

We recall that the standard approach to Nernst's theorem involves heat capacities and runs e.g. as in 2324 . We further limit ourselves to recall that black hole thermodynamics has some very peculiar features that make it special with respect to "standard" thermodynamics ( see e.g. 2526 ).

\section{Black holes branches as $T \rightarrow 0^{+}$}

In this section we discuss black hole thermodynamic branches near $T=0$. We first recall that for the entropy, as a function of $T, M, Q$, there is a branching into two different functions. In fact, although the entropy is a continuous function of $M, J, Q$, there are points such that the state equation $T(M, J, Q)$ cannot be inverted in order to get $M(T, J, Q)$. It happens that $\partial T / \partial M=0$ can be satisfied on suitable submanifolds, where standard conditions for the implicit function theorem fail. As a consequence, one can invert $T$ and obtain $M(T, J, Q)$, to be substituted into $S(M, J, Q)$ only away from these submanifolds, on the two branches $\partial T / \partial M>0$ and $\partial T / \partial M<0$, which are associated with the zero temperature limits obtained as finite mass extremal limit and as infinite mass limit respectively. In particular, for the same value of the variables $T, J, Q$ it is possible to get two different values of $S$. This is a sufficient reason for a multi-branching in the $S-T$ plane. These branches describe different systems. ${ }^{b}$ We first consider the finite mass extremal limit and then the infinite mass limit.

\subsection{Black hole extremal limit $M<+\infty$}

The violation of the third law in black hole thermodynamics as the extremal boundary is approached is well-known 3 . For the sake of completeness, we show that (1)

${ }^{\mathrm{b}}$ Points where $\partial T / \partial M=0$ correspond to critical submanifold points where $C_{Q J}$ diverges and changes sign. It has been proposed that a second order phase transition takes place there 3 . 
fails in the general case of a Kerr-Newman black hole. Let us define

$$
\begin{aligned}
& M_{E}^{2} \equiv \frac{1}{2}\left(Q^{2}+\sqrt{Q^{4}+4 J^{2}}\right) \\
& M_{N}^{2} \equiv \frac{1}{2}\left(Q^{2}-\sqrt{Q^{4}+4 J^{2}}\right)<0 ;
\end{aligned}
$$

$M_{E}^{2}, M_{N}^{2}$ are the roots of the equation $\left(M^{2}\right)^{2}-Q^{2} M^{2}-J^{2}=0$ and $M_{E}^{2}$ corresponds to the squared mass of the extremal Kerr-Newman solution having charge $Q$ and angular momentum $J$. It is useful to explicit the following relations between the above roots and the charge $Q$ and the angular momentum $J$ of the black hole: $Q^{2}=M_{E}^{2}+M_{N}^{2}, J^{2}=-M_{E}^{2} M_{N}^{2}$. Moreover, the difference $\left(M_{E}^{2}-M_{N}^{2}\right)$ is related to the area of the extremal solution for given values of $Q, J$ by $A_{E}=4 \pi\left(M_{E}^{2}-M_{N}^{2}\right)$. We can rewrite

$$
\begin{aligned}
& T=\frac{M}{2 \pi} \frac{\left(\left(M^{2}-M_{E}^{2}\right)\left(M^{2}-M_{N}^{2}\right)\right)^{1 / 2}}{\left(M^{2}+\left(\left(M^{2}-M_{E}^{2}\right)\left(M^{2}-M_{N}^{2}\right)\right)^{1 / 2}\right)^{2}-M_{E}^{2} M_{N}^{2}} \\
& S=\frac{\pi}{M^{2}}\left(\left(M^{2}+\left(\left(M^{2}-M_{E}^{2}\right)\left(M^{2}-M_{N}^{2}\right)\right)^{1 / 2}\right)^{2}-M_{E}^{2} M_{N}^{2}\right) .
\end{aligned}
$$

It is easy to show that, near the extremal states $M^{2} \sim M_{E}^{2}$, one has $M^{2}=M_{E}^{2}+$ $4 \pi^{2} M_{E}^{2}\left(M_{E}^{2}-M_{N}^{2}\right) T^{2}+\cdots$. Then, for $T \rightarrow 0^{+}$, one finds $S(T, Q, J) \sim \pi\left(M_{E}^{2}-\right.$ $\left.M_{N}^{2}\right)+4 \pi^{2} M_{E}\left(M_{E}^{2}-M_{N}^{2}\right) T+\cdots$, thus

$$
\begin{aligned}
\lim _{T \rightarrow 0^{+}}\left(S\left(T, Q_{1}, J_{1}\right)-S\left(T, Q_{2}, J_{2}\right)\right) & =\frac{1}{4}\left(A_{1 E}-A_{2 E}\right) \\
& =\pi\left(\sqrt{{Q_{1}}^{4}+4{J_{1}}^{2}}-\sqrt{{Q_{2}}^{4}+4 J_{2}^{2}}\right) .
\end{aligned}
$$

As expected, the difference in entropies is proportional to the difference of the areas of the corresponding extremal solutions, which depend on the macroscopic parameters. The limit (2) is to be intended as a right limit as $T \rightarrow 0^{+}$. The discussion which is developed in sect. [6] shows that it could be physically improper to assign by continuity a value to the entropy on the boundary $T=0$ of the thermodynamic manifold, i.e., it could be improper to assign the value $S_{E}=A_{E} / 4$ to extremal states.

The failure of $(\mathrm{N})$ implies that $(\mathrm{U})$ cannot be intended as absence of adiabatic transformations reaching $T=0$. It is interesting to notice that concavity (hypothesis a)) fails, as it is well-known, in black hole thermodynamics 27. In the black hole case, there exist curves approaching $T=0$ such that $C_{x}>0$ and other such that $C_{y}<0$. The existence of paths with $C_{x}<0$ allowing to approach $T=0$ is evident in the Kerr case 3 , where $C_{\Omega}<0$ and $C_{J}>0$ near the extremal limit. Cf. fig. 2] where the corresponding curves in the thermodynamic domain are shown.

In the general Kerr-Newman case, near the extremal state the heat capacity $C_{J, Q}$ is positive and goes to zero as $T$ at the extremal limit 3 . Other heat capacities at constant deformation parameters can be taken into account [27, (e.g. $C_{\Phi, Q}=$ $\left.C_{J, \Omega} ; C_{\Omega, Q}, C_{J, \Phi}, C_{\Omega, \Phi}\right)$. Some of them can be negative near $T=0$. Note that, 


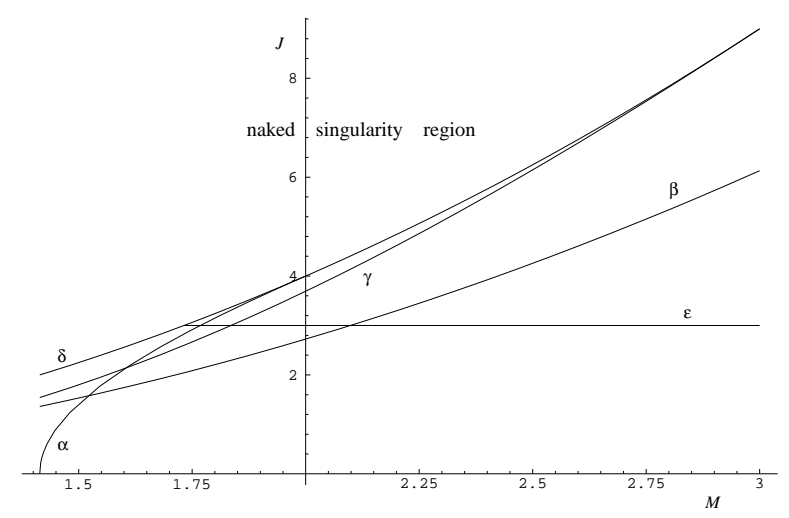

Fig. 2. In the Kerr case, the isentrope $\alpha: J=\sqrt{8 M^{2}-16}$, the line $\epsilon: J=$ const. $=3$, and the line $\gamma: J=\left(6 M^{3}\right) /\left(M^{2}+9\right)$, which corresponds to $\Omega=$ const. $=1 / 6$, are shown. Extremal states lie on the line $\delta: J=M^{2}$, above which naked solutions are found. The isentrope $\alpha$ ends at $M=2$, where it is tangent to the extremal manifold (see also Appendix A). Above the line $\beta: J=\sqrt{2 \sqrt{3}-3} M^{2}$ the heat capacity $C_{J}$ is positive. The line $J=\left(6 M^{3}\right) /\left(M^{2}+9\right)$ intersects the extremal manifold for $M=3$ (tangent to it).

however, the presence of paths (in the thermodynamic space) with negative heat capacity is in general not sufficient to ensure the violation of $(\mathrm{N})$. It only allows to de-link the violation of $(\mathrm{N})$ from the validity of $(\mathrm{U})$, in fact the non-uniformity of the sign of heat capacities near $T=0$ can allow adiabatic paths reaching $T=0$. See also Appendix C and 20 .

\subsection{The branch $T \rightarrow 0^{+}$for $M \rightarrow \infty$}

In black hole thermodynamics another limit of zero temperature is sometimes considered 328 . It is the limit as $M \rightarrow \infty$ e.g. in the Schwarzschild case. In fact, $T \sim 0$ only near the extremal states or for very large masses. But the latter limit cannot be considered on the same footing as the limit where extremal states are approached, indeed it is physically related to an unattainability principle in a straightforward way. No infinite mass can be allowed on physical grounds, whereas no hindrance to consider e.g. $Q^{2}=M^{2}$ in the Reissner-Nordström case is a priori given. However, an astrophysical black hole represents from a thermodynamic point of view a system reaching temperatures even much lower than the ones involved in experiments of low temperature physics and in actual experimental validation of $(\mathrm{N})$ and, moreover, it is interesting to stress that black hole thermodynamics allows to get systems having a very low temperature and a huge entropy in contrast with the low temperature behavior of standard systems. In particular, Planck's postulate $S \rightarrow 0$ for $T \rightarrow 0^{+}$is to some extent maximally violated ${ }^{\mathrm{c}}$. It is also interesting to show

${ }^{\mathrm{c}}$ We remark that the violation of $(\mathrm{N})$ in presence of diverging deformation parameters can occur in the case of systems for which $(\mathrm{N})$ holds at finite deformation parameters. See also 20 . 
that $(\mathrm{N})$ is violated in the sense that (11) fails in the case of a charged black hole. Of course, such a violation is impossible in the case of a Schwarzschild black hole, due to its too constrained thermodynamic phase space. Instead, let us first consider the Reissner-Nordström case; in the limit $M \rightarrow+\infty$ one can invert explicitly the relation between $M$ and $T$

$$
M^{2} \sim \frac{1}{64 \pi^{2} T^{2}}-8 \pi^{2} Q^{4} T^{2}
$$

then

$$
S(T, Q) \sim \frac{1}{16 \pi T^{2}}-2 \pi Q^{2}+O\left(T^{2}\right)
$$

and

$$
S\left(T, Q_{1}\right)-S\left(T, Q_{2}\right) \rightarrow 2 \pi\left(Q_{2}^{2}-Q_{1}^{2}\right) .
$$

See fig. 3. Obviously $S \rightarrow \infty$. If one considers the heat capacity $C_{Q}$ corresponding to the process under consideration, one finds that $C_{Q}<0$ so that the process involves the thermally unstable branch of black hole thermodynamics. We have just shown that $(\mathrm{N})$ fails but for a self-evident reason a sort of $(\mathrm{U})$ is automatically ensured. We stress again that (U) is not to be intended as the impossibility to reach $T=0$ in a finite number of processes in this case, but simply as the impossibility to get an infinitely massive black hole. (U) amounts to (U4), the most general notion of unattainability of the absolute zero.

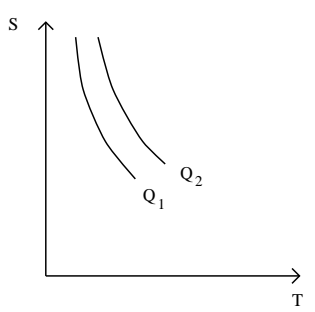

Fig. 3. Violation of $(\mathrm{N})$ in the large $-M$ limit. The qualitative behavior of the entropy is displayed for two different value of the black hole charge. Two isentropic lines are qualitatively displayed.

The general Kerr-Newman case can be treated analogously. The starting point is a large mass expansion of the equation of state for $T$

$$
T=\frac{1}{8 \pi} \frac{1}{M}-\frac{1}{128 \pi}\left(4 J^{2}+Q^{4}\right) \frac{1}{M^{5}}-\frac{1}{128 \pi} Q^{2}\left(4 J^{2}+Q^{4}\right) \frac{1}{M^{7}}+O\left(\frac{1}{M^{9}}\right) ;
$$

by inverting one finds

$$
\frac{1}{M}=8 \pi T+2048 \pi^{5}\left(4 J^{2}+Q^{4}\right) T^{5}+131072 \pi^{7} Q^{2}\left(4 J^{2}+Q^{4}\right) T^{7}+O\left(T^{9}\right)
$$

and

$$
S\left(T, Q_{1}, J_{1}\right)-S\left(T, Q_{2}, J_{2}\right)=2 \pi\left(Q_{2}^{2}-Q_{1}^{2}\right)
$$




$$
+48 \pi^{3}\left(4\left(J_{2}^{2}-J_{1}^{2}\right)+\left(Q_{2}^{4}-Q_{1}^{4}\right)\right) T^{2}+O\left(T^{4}\right) \cdot(5)
$$

We can deduce that, if the black hole is uncharged but rotating, $(\mathrm{N})$ is satisfied because $S\left(T, J_{1}\right)-S\left(T, J_{2}\right) \sim 192 \pi^{3}\left(J_{2}^{2}-J_{1}^{2}\right) T^{2} \rightarrow 0$ for $T \rightarrow 0^{+}$. So there is evidence in favor of the validity of $(\mathrm{N})$ in the case of uncharged rotating black holes of the Kerr family on the thermally unstable large mass branch of black hole thermodynamics (again, $C_{J}<0$ for $M \rightarrow \infty$ ). Only the presence of the charge is actually associated with the failure of $(\mathrm{N})$ on this branch. Thermal instability is verified also in the general Kerr-Newman case

$$
C_{J, Q} \sim-\frac{1}{8 \pi T^{2}}-96 \pi^{3}\left(4 J^{2}+Q^{4}\right) T^{2}+\cdots<0 .
$$

We find a behavior that is remarkable also from a thermodynamic point of view. In fact, the validity of (U4) can give rise both to $(\mathrm{N})$ and to the failure of $(\mathrm{N})$ 14. States at $T=0$ on this branch of black hole thermodynamics are evidently unphysical and disconnected from the finite mass states, we have a discontinuity which is directly related to the unavailability of an infinite energy which would be necessary in this case in order to obtain a zero temperature state (and an infinite entropy state). ${ }^{\mathrm{d}}$

It is also possible to find thermodynamic transformations joining together the extremal limit and the large mass limit to the zero temperature. One can choose e.g.

$$
J^{2}=M^{2}\left(M^{2}-Q_{0}^{2}\right) \tanh ^{2}\left(\frac{M}{M_{0}}\right)
$$

where $M_{0}, Q_{0}$ are constants. It is evident that the extremal limit is implemented as $M \rightarrow \infty$. In general, the extremal limit can be approached only asymptotically along these transformations and $(\mathrm{U})$ is preserved as above.

Note that, even in the charged case, one finds

$$
\lim _{T \rightarrow 0^{+}} \frac{S\left(T, Q_{1}\right)}{S\left(T, Q_{2}\right)}=1 .
$$

$(\mathrm{N})$ in some sense holds at the leading order but fails in the charged case when the difference is taken because of sub-leading terms depending on the charge $Q$, as it can be inferred from (3i), (4).

\footnotetext{
${ }^{\mathrm{d}}$ It is also remarkable that, if in general the entropy diverges for $T \rightarrow 0^{+}$, the axis $T=0$ acts as a vertical asymptote for the graph of $S$ in the $S-T$ plane and the possibility to find an adiabat reaching $T=0$ evidently is missing. The divergence of the entropy as $T \rightarrow 0^{+}$suggests that (U) holds (an isentropic at $S=+\infty$ appears to be unphysical). $T=0$ has to be excluded from the physical domain of $S$. Moreover, if this infinite entropy in the zero temperature limit can be obtained at the cost of an infinite energy, as in the black hole case, then there is also a (somehow trivial) physical reason for $(\mathrm{U})$.
} 


\section{Extremal black holes}

We shortly re-analyze the status of extremal black holes in the framework of black hole thermodynamics. The following considerations don't depend on taking a limit as $T \rightarrow 0^{+}$of the Smarr formula 29 , even if they are consistent with such a limit. Our point is that, in the case a discontinuity between non-extremal and extremal states occurs, such a limit makes no sense. Then, can be useful to re-analyze the extremal black holes from this point of view. Extremal black holes belonging to the Kerr-Newman family are characterized by the extremality constraint

$$
M^{2}=Q^{2}+\frac{J^{2}}{M^{2}}
$$

In the case of extremal black holes, the constraint equation (6) is equivalent to the (known) fundamental relation specifying the black hole state:

$$
M(Q, J)=\frac{1}{\sqrt{2}} \sqrt{Q^{2}+\sqrt{Q^{4}+4 J^{2}}}=M_{E}
$$

$M(Q, J)$ is a quasi-homogeneous function of degree $\frac{1}{2}$ and weights $(1 / 2,1)$ (see 3031 for a definition of quasi-homogeneous function):

$$
\frac{1}{2} \frac{\partial M}{\partial Q} Q+\frac{\partial M}{\partial J} J=\frac{1}{2} M=\frac{1}{2} \Phi_{\mathrm{b} h}^{\mathrm{e} x t r} Q+\Omega_{\mathrm{b} h}^{\mathrm{e} x t r} J,
$$

where $\Omega_{\mathrm{b} h}^{\mathrm{e} t r}, \Phi_{\mathrm{b} h}^{\mathrm{e} x t r}$ are the extremal black hole angular velocity and electric potential respectively. This quasi-homogeneity property is shared with Smarr formula $M(S, Q, J)$ for non-extremal black holes [in the latter case, one has a quasihomogeneous function of degree $1 / 2$ and weights $(1,1 / 2,1)]$. By differentiating (7) it is easy to show that along extremal states

$$
d M=\Omega_{\mathrm{b} h}^{\mathrm{e} x t r} d J+\Phi_{\mathrm{b} h}^{\mathrm{e} x t r} d Q
$$

This means that the extremal submanifold is an integral manifold for the Pfaffian form $\delta Q_{\text {rev }} \equiv d M-\Omega_{\mathrm{b} h} d J-\Phi_{\mathrm{b} h} d Q$, i.e., it is an adiabatic submanifold. This rephrasing is important for the discussion which follows in sect. 6.

It is also interesting to note that the area for an extremal black hole can be expressed as

$$
A_{\mathrm{e} x t r}=4 \pi\left(r_{+}^{2}+\frac{J^{2}}{M^{2}}\right)_{\mathrm{e} x t r}=4 \pi \sqrt{Q^{4}+4 J^{2}} .
$$

From (8]) one gets

$$
(d A)_{\mathrm{e} x t r}=\frac{8 \pi}{\sqrt{Q^{4}+4 J^{2}}}\left(Q^{3} d Q+2 J d J\right)=\frac{32 \pi^{2}}{A}\left(Q^{3} d Q+2 J d J\right) .
$$

The extremality constraint does not implies that $(d A)_{\mathrm{extr}}$ vanishes, and along extremal states in the case of Reissner-Nordström and Kerr black holes it is impossible to get $d A_{\mathrm{e} x t r}=0$ (i.e. the equation $A_{\mathrm{e} x t r}=$ const. is satisfied only for $M^{2}=Q^{2}=$ 
const. and $M^{4}=J^{2}=$ const. respectively), whereas for non-extremal black holes it is possible to get solutions for $d A=0$. Solutions of $d A_{\mathrm{e} x t r}=0$ are instead allowed in the Kerr-Newman case. See also Appendix A herein.

\section{Carathéodory's approach and the surface $T=0$.}

The approach of Carathéodory to thermodynamics identifies the the infinitesimal heat exchanged reversibly $\delta Q_{r e v}$ with an integrable Pfaffian form. See also 32 , where an original approach based on homogeneity symmetry is developed for standard thermodynamics 32 , and 3031 , where quasi-homogeneity symmetry replaces homogeneity in the case of black holes and of some self-gravitating matter systems. The Pfaffian form for black holes of the Kerr-Newman family is $\delta Q_{r e v} \equiv d M-\Omega d J-\Phi d Q$ and it is a non-singular integrable Pfaffian form defining a foliation of the thermodynamic manifold by means of the solutions of the Pfaffian equation $\delta Q_{\text {rev }}=0$. This one-form is smooth on the non-extremal submanifold, and it is continuous everywhere. Its integrability means that, in the inner part of the thermodynamic manifold (non-extremal states) $\delta Q_{r e v} \wedge d\left(\delta Q_{r e v}\right)=0$ is verified 30 . Carathéodory's approach to thermodynamics allows also to understand better the status of the surface $T=0$ both in standard thermodynamics 19 and in black hole thermodynamics. We limit ourselves to discuss the latter aspect herein. It is known that, according to Frobenius theorem, with a suitably regular integrable Pfaffian form is associated a foliation of the manifold into disconnected codimension one integral submanifolds. If one excludes the extremal boundary from the thermodynamic manifold, one finds that the leaves associated with $\delta Q_{\text {rev }}$ are the manifolds $A=$ const., i.e., $S=$ const. On this restricted manifold, the adiabatic inaccessibility property holds, which means that in the neighborhood of any point $\mathrm{P}$ there exists an infinite number of points which cannot be reached from $\mathrm{P}$ along solution curves of $\delta Q_{\text {rev }}=0$ [this property, introduced by Carathéodory (see ${ }^{15}$ ), is equivalent to the integrability property]. Then, for $T>0$ the integral manifolds of the Pfaffian form $\delta Q_{\text {rev }}$ are the surfaces $S=$ const. Given any non-extremal state, any path solving the equation $\delta Q_{\text {rev }}=0$ in the thermodynamic manifold has to lie on a isentropic surface.

The extremal submanifold is very peculiar. In fact, the surface $T=0$, which corresponds to the extremal submanifold, is an integral manifold of the Pfaffian form $\delta Q_{\text {rev }}$, in the sense that it solves the equation $\delta Q_{\text {rev }}=0$, as we have seen in sect. 5] It could be considered naively as a leaf, but the lack of some regularity properties of $\delta Q_{\text {rev }}$ on the extremal submanifold has important consequences. Let us consider the Reissner-Nordström case. By posing $M^{2}=x ; Q^{2}=y$ one finds

$$
\delta Q_{\text {rev }}=\frac{1}{2 \sqrt{x}} d x-\frac{1}{2(\sqrt{x}+\sqrt{x-y})} d y
$$


where $y \leq x$. Given a black hole state $\left(x_{0}, y_{0}\right)$, the states which are adiabatically reachable from it lie on the curves that are solutions of the following Cauchy problem

$$
\begin{aligned}
& \frac{d y}{d x}=1+\sqrt{1-\frac{y}{x}} \\
& y\left(x_{0}\right)=y_{0} .
\end{aligned}
$$

The solution of this problem exists and it is unique for any initial non-extremal state; moreover, it corresponds to the standard isoareal ${ }^{\mathrm{e}}$ solution. If, instead, one considers an extremal state as initial point, the Cauchy problem

$$
\begin{aligned}
& \frac{d y}{d x}=1+\sqrt{1-\frac{y}{x}} \\
& y\left(x_{0}\right)=x_{0}
\end{aligned}
$$

allows two solutions:

$$
y(x)=x,
$$

which means that the extremal states are adiabatically connected each other, and the solution

$$
y(x)=2 \sqrt{x_{0}} \sqrt{x}-x_{0}
$$

which holds for $x \in\left(x_{0} / 4, x_{0}\right]$ and means that extremal states are also adiabatically connected to non-extremal ones. The key-point is that on the extremal manifold, the right member of the differential equation (14) is no more smooth (actually, it is not $C^{1}$ and even the weaker Lipschitz condition is not satisfied). ${ }^{\mathrm{f}}$ This is a serious problem from a thermodynamic point of view, because the adiabatic inaccessibility is jeopardized by the $T=0$ manifold. It seems indeed to be possible to reach adiabatically any non-extremal state from any other one by passing through extremal states (which are non-isoareal). This would imply a failure of the second law of thermodynamics. In fact, it would be possible to find a Carnot cycle having thermal efficiency equal to one, against Ostwald's statement of the second law. In other terms, it would be possible to transform heat entirely into work, against the second law. On this topic, see a discussion in sect. 6.1 Moreover, because of the intersection of integral manifolds, even if only at $T=0$, one cannot conclude that there is a foliation of the whole thermodynamic manifold [extremal manifold included] but what one could define an almost-foliation, i.e. a foliation except for a zero measure set (the integral manifold $T=0$ ).

From a physical point of view, in order to avoid the above singular behavior of the

\footnotetext{
eThe adjective "isoareal" is coined in agreement with the standard coining of adjectives in thermodynamics (see e.g. isothermal,isochoric,...). An isoareal transformation is a transformation in which each state has the same area: "isoareal = with the same area".

${ }^{\mathrm{f}}$ Without solutions like 16, one could conclude that the extremal manifold is a leaf of the thermodynamic manifold. One is instead forced to introduce a discontinuity in order to obtain a well-behaved foliation of the whole thermodynamic manifold.
} 
thermodynamic foliation, one could decide that the surface $T=0$ should be a leaf itself, that is, to exclude the set of solutions (16) for the above Cauchy problem. Notice that the set of solutions (16) corresponds to the isoareal solutions $d A=0$. Obviously, the existence of extremal black holes which have the same area as nonextremal states is not questioned; what is questioned by refusing the set of solutions (16) is the validity of $S \propto A$ also in the case of extremal black holes. The geometric foliation of the whole black hole manifold whose leaves are given by $A=$ const. should correspond to the thermodynamic foliation only in the case of non-extremal states.

In order to avoid problems with thermodynamics, one could construct a foliation of the thermodynamic manifold whose leaves are

the surfaces $S=A / 4=$ const. for non-extremal states

the surface of extremal states.

The leaves for the non-extremal manifold are the usual ones, which can be generated by means of the Pfaffian form $\delta Q_{r e v}$. Instead, the exceptional integral manifold $T=$ 0 is assumed to be isentropic and such that any extremal state has an entropy which is different from the one of any near extremal state (or, by continuity, one could pass adiabatically from non-extremal states to extremal ones). The Bekenstein-Hawking law ensures that $S=A / 4$ can assume arbitrarily large values and, a priori, also very small values, the only lower bound could be given by the onset of a quantum gravity regime (which occurs in the limit $M \rightarrow 0$ ). Without considering such small values of $S$ implying a quantum gravity regime, it is reasonable, on a purely thermodynamic footing, to assume that $S_{E}=0$ for any extremal state. A further discussion is found in the following.

As a consequence of the latter assumption, the thermodynamic foliation one obtains is given by the following discontinuous entropy $S(M, Q, J)$ :

$$
\begin{aligned}
S(M, Q, J) & =\frac{A}{4} \quad \text { for non-extremal states } \\
& =0 \quad \text { for extremal states. }
\end{aligned}
$$

About the thermodynamic consistency of this assumption, and its relation with $(\mathrm{U})$, see also the discussion in sect. 7.1 herein.

\subsection{The Bekenstein-Hawking formula for extremal states and the Carnot-Nernst cycle}

We discuss in detail the problems that can arise if the above failure of the adiabatic inaccessibility property is verified. Let us consider a thermal Carnot cycle in the plane $T-S$ (see. fig. 4 the cycle is clockwise), having the lower isotherm exactly at $T=0$. We define it as Carnot-Nernst cycle.

If it is possible to perform a Carnot-Nernst cycle, then one can be able to construct a thermal machine with efficiency exactly equal to one, in violation of the 


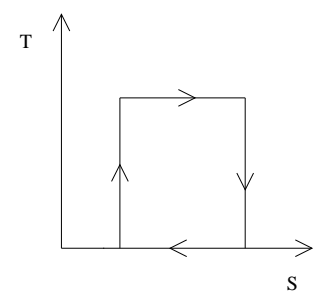

Fig. 4. Carnot-Nernst cycle involving the isotherm at $T=0$. Its efficiency is one, against the second law.

second law of thermodynamics. This argument is substantially due to Nernst, who introduced it for supporting the third law of thermodynamics (see e.g. 1311415).

Of course, one has to check the actual possibility to perform such a cycle. The following hypotheses are taken into consideration:

$\alpha$ ) extremal states can be reached by means of reversible adiabatic paths by starting from non-extremal states;

$\beta$ ) reversible transformations along extremal states discussed in sect. 5. are allowed;

$\gamma)$ non-isoareal transformations along extremal states exist;

$\delta$ ) the Bekenstein-Hawking law holds for extremal states.

If the hypotheses $\alpha), \beta), \gamma$ ) and $\delta$ ) are all verified, then the Carnot-Nernst cycle can be performed and a violation of the second law occurs. We discuss now the above hypotheses.

Hypothesis $\alpha$ ) is verified, in the light of the existence of integral manifolds of $\delta Q_{\text {rev }}$ which are allowed to reach $T=0$, unless some discontinuity occurs.

Hypothesis $\beta$ ) is more critical. In the case of standard thermodynamics objections against the possibility to perform a reversible transformation at $T=0$ have been raised 33 , because of the impossibility to improve a change between the adiabatic constraint used in approaching $T=0$ and the adiabatic constraint in performing the adiabatic isotherm $T=0$. The possibility to perform of transformations along $T=0$ states in standard thermodynamics has been criticized by Einstein 34 (cf. also 1114 ), both from the point of view of the unavoidable presence of nonnegligible irreversibility occurring near the absolute zero, and from the point of view of the actual possibility to perform an ideal transformation along $T=0$. Actually, e.g. in the case of a Reissner-Nordström black hole, one could effectively distinguish between the adiabat approaching the extremal states (which is characterized by the equation $Q^{2}=2 M r_{+}-\left(r_{+}\right)^{2}$, where $r_{+}$is the radius of the initial black hole state), and the adiabat along extremal states (whose equation is $Q^{2}=M^{2}$ ). Rejecting a priori $\beta$ ) would mean implicitly to introduce a "discontinuity" for thermodynamics in the behavior of extremal states with respect to non-extremal ones. The impossibility to perform any transformation along the $T=0$ isothermal surface would be surely peculiar, in the sense that it is a property which distinguishes the $T=0$ 
submanifold with respect to the thermodynamic space at $T>0$.

Moreover, one has to ensure that transformations such that $d A<0$ along the extremal submanifold can occur in order to implement the Carnot-Nernst cycle in black hole thermodynamics. We have found no definitive counter-example against $d A<0$ along extremal states.

Hypothesis $\gamma$ ) is introduced because the existence of non-isoareal transformations along extremal states is required if one has to perform the Carnot-Nernst cycle. $\gamma$ ) is verified, because $d A \neq 0$ is allowed along extremal states (see sect. 5).

If we assume that also hypothesis $\delta$ ) holds, then then a violation of the second law in a thermostatic framework occurs. As a consequence, we look for solutions. Moreover, whichever doubt one could raise against the possibility to perform the Carnot-Nernst cycle, one can agree with Einstein's statement that the existence of adiabatic paths allowing a Carnot-Nernst cycle is "very hurtful to one's physical sensibilities" 34.

A possibility to avoid the failure of the second law implied by the CarnotNernst cycle consists in rejecting $\alpha$ ) in the frame of black hole thermodynamics, by requiring that a discontinuity does not allow to perform the adiabats and reach the extremal states (even if non-extremal states are dense near the extremal ones along adiabats). ${ }^{\mathrm{g}}$ Notice that the failure of the Bekenstein-Hawking law for extremal states implements such an impossibility to attain along isentropes the extremal states. Then a failure of hypothesis $\delta$ ) implies a failure of $\alpha$ ), and the failure of hypothesis $\delta$ ) is a natural solution to the above problem. We show in the following subsection that the failure of $\delta$ ) is less unnatural than a first look could suggest, because a discontinuous behavior of thermodynamics is in any case verified.

\subsection{Discontinuity between extremal states and non extremal ones. a thermostatic frame analysis and the failure of $S=A / 4$}

We show that, both if the Bekenstein-Hawking law is verified and if it is not verified along extremal states, one is forced to admit that thermodynamics does not behave continuously in passing from non-extremal states to extremal ones. If the Bekenstein-Hawking law is violated, the discontinuity is evident. If, instead, the Bekenstein-Hawking law is maintained also in the extremal case, it is anyway true

\footnotetext{
${ }^{\mathrm{g}}$ In the frame of standard thermodynamics, Nernst invokes the failure of hypothesis $\alpha$ ) in the sense of the absence of adiabats reaching $T=0$, whereas in black hole thermodynamics one can invoke (U) only in order to protect the second law (obviously the equivalence between (U) and $(\mathrm{N})$ is violated). In 1315, the Carnot-Nernst cycle is utilized with the aim to justify the following principle: The zero temperature states are so poorly populated that it is impossible to draw a continuous line between them. This point of view introduces actually an element of topological difference between states at $T>0$ and states at $T=0$, a "discontinuity" in thermodynamics, unless one does not postulates that the density of states starts decreasing at a positive $T$ near enough to the absolute zero (this requirement could match Munster's statements about the failure of thermodynamics for finite-size systems sufficiently near $T=023$ ). This poor population density does not seem to affect extremal black holes.
} 
that a discontinuity is verified, because reversible transformations along extremal states are always adiabatic, as seen in sect. 5 and in general non-isoareal (cf. also Appendix A), contrarily to what happens for non-extremal states, where adiabatic reversible transformations are necessarily isoareal.

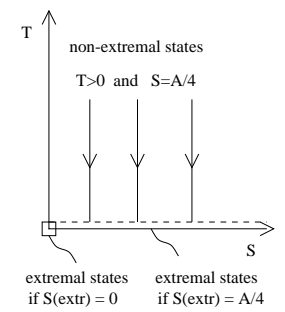

Fig. 5. $T-S$ plane in black hole thermodynamics. Three isentropes approaching extremal states are shown. The dashed line near $T=0$ indicates that a discontinuity in thermodynamics appears both in the case that $S=A / 4$ holds for extremal states and in the case a different law is implemented (the case $S=0$ is displayed by means of a small box).

In the light of the hypothesis of failure for the Bekenstein-Hawking law along extremal states, we discuss shortly what happens from a statistical mechanical point of view. Extremal black hole entropy can be, in line of principle, valued by means of quantum mechanics: The Von Neumann entropy of extremal black holes is a priori calculable and it is the only meaningful entropy that can be associated with a state by studying it at exactly $T=0$, without considering a limiting process as $T \rightarrow 0^{+}$. A dichotomy between the limit as $T \rightarrow 0^{+}$of the thermodynamic entropy and the $T=0$ entropy for extremal states appears in 35 and in a paper concerning the quantization of extremal Reissner-Nordström black holes 36 . Zero entropy is found by working separately on extremal states, a non-vanishing entropy is allowed if a limiting process starting from a quantization of non-extremal states is given rise 36 . The same dichotomy is implicit in $\frac{79}{\text {, }}$, where $S=0$ for extremal states and $S=A / 4$ for any non-extremal black hole ${ }^{\mathrm{h}}$. Doubts against limiting processes for calculating the entropy of extremal black holes are raised also in 36 (therein and in $[35$ interesting comments about the results obtained for BPS states in string theory approach are found too). In black hole thermodynamics, one is suggested to introduce a discontinuity of thermostatics between $T>0$ and the absolute zero. A discontinuity is appealing in black hole thermodynamics because a very different physics is involved in the case of extremal black holes with respect to non-extremal ones (see e.g. 7937386 ). From this point of view, one has also to take into account that, although a continuous behavior of some geometrical properties

\footnotetext{
${ }^{\mathrm{h}}$ In statistical mechanics, there are subtleties related with the order in which the limit as $T \rightarrow 0^{+}$ and the thermodynamic limit are taken in the calculation; the correct procedure consists in taking first the thermodynamic limit and then the limit as $T \rightarrow 0^{+}$. This further problem does not seem to be relevant for black holes.
} 
is verified, there are important differences in properties like the topology of the manifold. The Euler characteristic changes and this topological difference has been related with the thermodynamic differences between extremal and non-extremal black holes, being the global thermodynamic functionals linked with the global properties of the manifold 788394041 .

In the following section, we relate in a thermostatic framework the unattainability property and the failure of the Bekenstein-Hawking law.

\section{Thermostatics, (U) and $S_{E}=0$}

The violation of $(\mathrm{N})$ near the extremal states $M<+\infty$ from a thermodynamic point of view does not forbid the attainment of the zero temperature state. In order to conciliate the validity of $(\mathrm{U})$ and the violation of $(\mathrm{N})$ a reasonable hypothesis is that near $T=0$ in black hole thermodynamics a (possibly abrupt) change in thermodynamic properties of the system occurs. We are inspired by Landsberg's hypothesis c), relative to a possible discontinuity ensuring (U) against the failure of $(\mathrm{N})$. It is very interesting, because in studying the implication $(\mathrm{U}) \Rightarrow(\mathrm{N})$ Landsberg not only postulates the validity of (U) but also he tries to retrace the possibility to get $(\mathrm{U})$ and not $(\mathrm{N})$ in a peculiar behavior of some thermodynamic functions. The infinite mass case shows that it is not strictly necessary such a behavior, because the attainment of $T=0$ is forbidden in that case simply by the first law (conservation of energy). In standard thermodynamics, when (N) holds, the attainment of the absolute zero is generally thought to be forbidden by the second law (impossibility of $\Delta S<0$ for an adiabatic process of a closed system). For the finite mass extremal case we have shown that there is the possibility to de-link $(\mathrm{U})$ from $(\mathrm{N})$ but we think it is interesting to investigate also if there are thermodynamic arguments suggesting (U) beyond the dynamic theorem of Israel 4 , whose thermodynamic implications are discussed in sect. 8 It is also useful to recall the potential relevance of $(\mathrm{U})$ in relation with the Cosmic Censorship Conjecture (CCC), the third law in the (U)

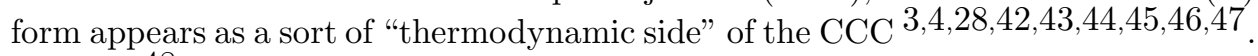
See also 48 .

In order to corroborate the hypothesis of a discontinuity in the sense of Landsberg, the most straightforward study involves the analysis of the behavior of "standard" adiabatic quantities near the extremal limit. If e.g. some adiabatic compressibility should vanish then the hypothesis would be verified, indeed it would be impossible to carry out the adiabat connecting a non-extremal state to an extremal one. Landsberg makes the example of an abrupt divergence in the elastic constants of a solid as a conceivable ideal process preventing a solid to reach a zero temperature state by means of quasi-static adiabatic volume variations (the hypothesis of 13 is compatible with the vanishing near $T=0$ of the (adiabatic) compressibilities that are related with elastic constants in ordinary thermodynamics; particularly, for standard systems one can define the compressibility modulus as the inverse of the compressibility; it is proportional to the Young modulus in the case of a solid). 
But our analysis does not show a peculiar behavior of adiabatic derivatives and does not suggest the kind of discontinuity characterizing Landsberg's hypothesis. Cf. also the appendix of 27 , where some adiabatic derivatives are calculated. ${ }^{\mathrm{i}}$

We now give a thermostatic argument in favor of the unattainability of extremal states. It is based on the assumption that extremal states have zero entropy.

\section{1. (U) and extremal states with $S_{E}=0$}

It is remarkable that, if extremal states have $S_{E}=0$, then one has a natural naive argument for implementing (U). Let us consider the following hypotheses:

s1) the system is composed by a non-extremal black hole and matter; it is insulated, i.e., no exchange of energy, both in form of heat and in form of work, is allowed. $^{\mathrm{j}}$

s2) Both the initial and the final state are equilibrium states (which can be a nontrivial postulate for states at $T=0$ ).

s3) The final state is an equilibrium state between the black hole and matter.

s4) $S_{E}=0$.

Hypothesis s1) means that the principle of increase of entropy holds for the system. It implies that energy exchanges with the rest of the universe are forbidden. In a collapse situation, there can be surroundings of the system matter+black hole whose entropy variation could play a role in the application of the second law of thermodynamics. Hypothesis s2) is natural, in the sense that the thermodynamic entropy is unambiguously defined only for equilibrium states. s3) and s4) are discussed below. We can consider at first the case in which all the matter can be used for making extreme the black hole. For the initial state one has contributions to the entropy from the matter and a non-extremal black hole (NE): $S_{t o t}^{i n}=S_{N E}^{i n}+S_{m a t}^{i n}$; in the final state one has only an extremal black hole, so that, according to s4), $S_{t o t}^{f i n}=S_{E}=0$. Clearly the second law requires for the adiabatic process $S_{t o t}^{f i n} \geq S_{t o t}^{i n}$, which is impossible in our case.

One can also relax the hypothesis that all the matter is used for making extreme the black hole; if the matter at the end is in equilibrium with the black hole according to hypothesis s3), then the second law would require $S_{t o t}^{f i n}=S_{m a t}^{f i n} \geq S_{N E}^{i n}+S_{m a t}^{i n}$, which for ordinary matter is again impossible $\left(S_{\text {mat }}^{\text {fin }} \sim 0\right.$ because of $\left.(\mathrm{N})\right)$. One could then arbitrarily approach an extremal state but the "jump" onto extremality would be forbidden by the second law.

The final state could also be an equilibrium state if at least a portion of the residual matter is kept thermally insulated with respect to the black hole (violation of

${ }^{i}$ Nevertheless, in a thermostatic frame, the analysis of stability properties for Kerr-Newman black holes can suggest the (U) property of extremal states and are carried out in Appendix D

${ }^{\mathrm{j} F o r}$ the principle of increase of entropy, it would be sufficient that the system is closed and undergoes an adiabatic transformation. 
condition s3)) by some external mean, in which case the final state should have a contribution $S_{\text {mat }}^{f i n}>0$. But it is hard to see how this framework could allow the attainability of extremal states without a violation of the second law. One should allow the formation of extremal states from non-extremal ones in such a way to preserve the principle of increase of the entropy for the thermodynamic universe under consideration $^{\mathrm{k}}$ (an highly non-trivial task in light of the fact that $S_{N E}^{i n}$ is a huge number in general).

This hypothesis relating (U) to the second law is to be compared with processes that allow to get extremal states. In general, they don't correspond to quasi-static processes. Extremal black hole formation from extremal collapsing thin shells 9 and from charged thin shell collapsing on a non-extremal black hole (see 45 ) are examples of these processes. Could one define their initial state as an equilibrium state? In the first example, the shell is pushed from infinity. In the second case, the shell has to be fired onto the non-extremal black hole. If the quantum gravity result $S_{E}=0$ is true, then a careful analysis of the second law is required in order to ensure that, at a deeper level with respect to the naive analysis for the aforementioned processes, the second law is actually preserved. Even if this analysis were essentially unmodified, a detailed analysis of stability could reveal that the probability of these processes is very low; in 46 is indeed underlined that the extremalization process by means of thin shells is highly unstable under perturbations (see the conclusions therein). Quantum effect could also play a relevant role in this case, as follows from 4950. In 51, it is conjectured $S_{E}=0$ by starting from quantum gravity considerations. It is interesting to note that there is consistency with our conjecture for implementing $(\mathrm{U})$ in a thermostatic context.

\section{2. $S_{E}=0$ and the merging of two extremal black holes}

We discuss a consistency check for the hypothesis $S_{E}=0$. Let us consider two black extremal black holes $\left(M_{1}, Q_{1}, J_{1}\right)$ and $\left(M_{2}, Q_{2}, J_{2}\right)$ (one variable, e.g. $M$, of course depends on the other two because of the extremal constraint). Let us allow the two extremal black holes to merge and that no energy is exchanged with the rest of the universe during the process [one could as well consider a more general situation in which the couple is thermally insulated, i.e., it does not exchange heat with its surroundings]. We suppose that the final state consists of a single black hole resulting from the merging of the two initial extremal states. We wonder if the final state could be extremal. The point is that, if the final state could be extremal, then a violation of the second law could still occur, in fact the process is irreversible and the final entropy should be greater than the initial one but, if the final state is extremal and no energy is exchanged with the rest of the universe, one would find

${ }^{k}$ For "thermodynamic universe" we mean the smallest closed and thermally isolated system of interest (e.g., in the case of a black hole and matter falling into it, if thermal and matter exchanges with the surroundings are impossible, the thermodynamic universe is the system black hole + matter). 
$S_{E 1}+S_{E 2}=S_{i n}=0=S_{E 12}=S_{f i n}$. Let us define, as in 27,

$$
a^{2}(M, Q, J) \equiv M^{4}-M^{2} Q^{2}-J^{2}
$$

from 27 we know that, by defining $a_{12}^{2} \equiv a^{2}\left(M_{1}+M_{2}, Q_{1}+Q_{2}, J_{1}+J_{2}\right)$, $a_{1}^{2} \equiv a^{2}\left(M_{1}, Q_{1}, J_{1}\right)$, and $a_{2}^{2} \equiv a^{2}\left(M_{2}, Q_{2}, J_{2}\right)$ one has

$$
\begin{aligned}
a_{12}^{2}-\left(a_{1}+a_{2}\right)^{2} & =\left(\frac{M_{2}}{M_{1}} a_{1}-\frac{M_{1}}{M_{2}} a_{2}\right)^{2}+\left(\frac{M_{2}}{M_{1}} J_{1}-\frac{M_{1}}{M_{2}} J_{2}\right)^{2} \\
& +2 M_{1} M_{2}\left[\left(M_{1}+M_{2}\right)^{2}-\left(Q_{1}+Q_{2}\right)^{2}\right] \\
& +2\left(M_{1} M_{2}-Q_{1} Q_{2}\right)\left(M_{1}^{2}+M_{2}^{2}\right) .
\end{aligned}
$$

We wish to see if it is possible that $a_{12}=0$, which would imply that the final state is extremal. In our case, the above formula simplifies because $a_{1}=0=a_{2}$ for the initial extremal states. Moreover, one has $M_{1}=M_{1 E}$ and $M_{2}=M_{2 E}$. Then one finds

$$
\begin{aligned}
a_{12}^{2} & =\left(\frac{M_{2 E}}{M_{1 E}} J_{1}-\frac{M_{1 E}}{M_{2 E}} J_{2}\right)^{2} \\
& +2 M_{1 E} M_{2 E}\left[\left(M_{1 E}+M_{2 E}\right)^{2}-\left(Q_{1}+Q_{2}\right)^{2}\right] \\
& +2\left(M_{1 E} M_{2 E}-Q_{1} Q_{2}\right)\left(M_{1 E}^{2}+M_{2 E}^{2}\right) .
\end{aligned}
$$

If $J_{1} \neq 0$ and/or $J_{2} \neq 0$, no matter which values one considers for $Q_{1}, Q_{2}$, then $a_{12}>0$ and the final state is a non-extremal state. The final entropy is surely much greater than the initial one. If $J_{1}=0=J_{2}$, then it is possible to find a final state which is still extremal if one merges two extremal Reissner-Nordsström black holes having charges with the same sign, as it is evident from

$$
\begin{aligned}
a_{12}^{2} & =2 M_{1 E} M_{2 E}\left[\left(M_{1 E}+M_{2 E}\right)^{2}-\left(Q_{1}+Q_{2}\right)^{2}\right] \\
& +2\left(M_{1 E} M_{2 E}-Q_{1} Q_{2}\right)\left(M_{1 E}^{2}+M_{2 E}^{2}\right) .
\end{aligned}
$$

One should question if it is possible to allow $S_{E}=0$ and to preserve the second law in a real process of merging. Notice that even a very small angular momentum would protect the second law.

We don't study here this problem, we limit ourselves to the above considerations. Of course, in light of the risk for violations of the second law, also the hypothesis s4), $S_{E}=0$ should be questioned.

\section{Irreversibility Frame}

We now discuss the meaning of $(\mathrm{U})$ as it is rigorously proved in 4 . It is important to stress that in Israel's proof (U) holds from a dynamic point of view. In particular, it is shown that a non-extremal black hole cannot become extremal in a finite advanced time if the accreted matter stress-energy tensor satisfies the weak energy condition in a neighborhood of the outer apparent horizon and remains bounded and continuous 4 . Israel's result shows that, along a continuous 
process, in a finite advanced time it is impossible to destroy the trapped surfaces, which are present in the non-extremal states and instead are missing in the extremal one. This implies that the process $(\mathrm{NE}) \rightarrow(\mathrm{E})$ from a non-extremal black hole $r_{+}^{N E}-r_{-}^{N E}>0 \Leftrightarrow k^{N E}>0$ to an extremal black hole $r_{+}^{E}-r_{-}^{E}=0 \Leftrightarrow k^{E}=0$ as a final state requires an infinite timel. In the following we stress that also the subclass of thermodynamic processes is constrained by Israel's result, at least as far as approximations of quasi-static processes are implemented by means of accretion of matter whose stress-energy tensor is bounded and continuous and satisfies the hypotheses of 4 . Approximations of quasi-static processes e.g. by means of point-like particles satisfy the aforementioned requirements if suitably corrected (they would imply a distributional stress-energy tensor that should be corrected by taking into account the finiteness of the Compton wavelength of the particles). Some dynamic restrictions in Israel's proof don't allow a full identification of such a result with the unattainability (U4) of the extremal states.

We show that Israel's result implies that extremal black holes cannot be considered as equilibrium states contiguous to non-extremal black hole equilibrium states. The thermodynamic manifold of equilibrium states has to present a discontinuity. The framework of irreversible thermodynamics is the most appropriate in order to include in thermodynamics Israel's dynamic information. In fact, irreversible thermodynamics allows the introduction of the notion of relaxation time to an equilibrium state, and gives the effective physical time-scale with respect to which a process can be considered properly as a good approximation of a quasi-static process. The inaccessibility in a finite time of the extremal states from non-extremal ones by means of a continuous process can be rephrased as the impossibility to carry on an approximate quasi-static process joining non-extremal states to extremal ones, due to the divergence, which is met in approaching extremal states, of the "relaxation" time to the equilibrium state (that corresponds to the formation time of the stationary black hole state). We corroborate this point of view as follows. In 4 is found that, in order to squeeze out trapped surfaces, it is necessary an infinite time $\tau_{\text {no trapped }}=\infty$. Since an extremal black hole has no trapped surfaces, it follows the third principle $\tau_{(N E) \rightarrow(E)}=\infty$. In the framework of irreversible black hole thermodynamics one can conclude that $\tau_{\text {relaxation }}$ to an extremal equilibrium state coincides with $\tau_{(N E) \rightarrow(E)}=\infty$ if the latter is relative to a generic dynamical bounded and continuous process as in 4 , even if a correspondence between relaxation phenomena and irreversible black hole thermodynamics still is missing. See however [52, in particular section 6.3.3 therein, where a time-scale $\tau \sim 1 / T_{\mathrm{b} h}$ is proposed for the decay of a perturbed black hole to a stationary state $52 \mathrm{~m}$. Aspects of irreversible black hole thermodynamics are also explored in $[53$ (where a forma-

\footnotetext{
${ }^{1}$ The process $(\mathrm{NE})_{1} \rightarrow(\mathrm{NE})_{2}$ between non extremal states $(\mathrm{NE})_{1}$ and $(\mathrm{NE})_{2}$ can occur in a finite time.

${ }^{m}$ Note that also the "mining process" time-scale of 44 is of the same order.
} 
tion time-scale again order of $1 / T_{\mathrm{b} h}$ appears) and in particular in 5455. We don't develop herein an irreversible thermodynamics formalism for black holes, which should be the subject of further investigations.

Israel's result implies that thermodynamic formalism involving quasi-static processes cannot be extended to the extremal states because, from a physical point of view, approximating ideal quasi-static processes by means of (roughly) very slow processes looses sense in processes involving a transition from near extremal states to extremal ones because of the infinity in the relaxation time. Equilibrium thermodynamic formalism gives rise to a consistent description of very slow processes only if the relaxation times of various parameters defining the equilibrium state are much bigger or much smaller than the measurement time. In the former case the the parameters get a constant value, in the latter they get their equilibrium value 16. The case of a measurement time of the same order as the relaxation time is critical 16. In the case of a black hole, a measurement time suitable in order to measure the reaching of a black hole equilibrium state should be much longer than the formation time. For the case of an extremal black hole state attained by means of a continuous process, there is no satisfactory measurement time because at best an infinite measurement time should be compared with an infinite relaxation (formation) time. This means also that there is an intrinsic inaccessibility of extremal states if they have to be reached by means of a continuous quasi-static process. Other considerations about the failure of thermodynamics near the extremal limit can be found in 17 .

We can now implement a better comparison of Landsberg's hypothesis c) with the actual behavior of black holes near the extremal limit as dictated by 4 . The infinity of the time required in order to get an extremal state suggests that the dynamic process meets some hindrance near the extremal state to be carried further on in a finite time, if the process is "continuous". Qualitatively, this is e.g. suggested by the fact that adding charge to a non-extremal Reissner-Nordström black hole becomes more and more difficult due to the increase in the electrostatic repulsion. The impossibility to increase the black hole charge/angular momentum in a finite time till the extremality condition is implemented just resembles Landsberg's suggestion 13 of a discontinuity near the absolute zero (violation also of the hypothesis c)), but it involves an intrinsically dynamical information (as the infinite time required in order to implement the process). The peculiar characteristic of black hole thermodynamics, from the point of view of the third principle, is that "infinity of processes" of the standard formulation for (U) is substituted with "infinity of time". Actually the latter formulation seems more general than the former, in the sense that "infinity of processes" can easily imply "infinity of time" (but obviously not vice versa).

A final comment can be made concerning (U) according to Israel and (U) as required by the second law in 7.1 Let us one assumes the following conjecture: The gravitational entropy of black holes is non-zero and it is given by Bekenstein- 
Hawking law only in presence of trapped surfaces. Then Israel's proof can be interpreted as the dynamics-based side of the thermodynamics-based hindrance to reach extremal states shown in 7.1 It is also tempting to remark that the aforementioned conjecture, together with the above thermodynamic analysis, can enforce the conjecture interpreting black hole entropy as "entanglement entropy" 56. In fact, the "entanglement entropy", which is obtained by tracing the von Neumann entropy over unavailable degrees of freedom, can be associated with a trapped surface (the trace should be taken over the quantum field modes contained in the trapped surface). Moreover, for an "entanglement entropy" satisfying the area law there is no reason why it should approach a constant (zero) value as the extremal states are approached ${ }^{\mathrm{n}}$, because the area of the trapping surface depends on geometric parameters and it does not vanish near the extremal states, no matter how near the extremal boundary the black hole could be. Even the discontinuity of $S$ at the extremal boundary could be justified (no trapped surface would mean zero entanglement entropy).

We don't want to claim that "entanglement entropy" is mandatory, we simply limit ourselves to note that it seems to have chances to match both the failure of $(\mathrm{N})$ and the validity of $(\mathrm{U})$ in black hole thermodynamics. The alternative view to consider the failure of $(\mathrm{N})$ as due to "frozen non-equilibrium states" in black hole thermodynamics does not seem to be plausible. See a discussion in Appendix B.

\section{Conclusions}

We have analyzed the third law of thermodynamics from the point of view of a purely thermodynamic framework. A particular reference to P.T.Landsberg ideas has been made. We have discussed both the branches as $T \rightarrow 0^{+}$one can find in thermodynamics. An analysis of the failure of the implication $(\mathrm{U}) \Rightarrow(\mathrm{N})$ in black hole thermodynamics has revealed a special status to (U) in black hole thermodynamics. The analysis of the limit $T \rightarrow 0^{+}$for large black hole masses, where $(\mathrm{U})$ is necessarily implemented, has shown that $(\mathrm{N})$ is satisfied if the black hole is uncharged. In the branch $M \rightarrow \infty$ the failure of (N) vs. the validity of (U4) in the charged case has to be considered as a particular property of black hole thermodynamics.

In the framework of Carathéodory's approach to thermodynamics, we have point out which kind of problems can arise if the Bekenstein-Hawking law holds also for extremal states. In particular, the possibility to get a Carnot-Nernst cycle has been pointed out. Being such a cycle involved in a violation of the second law of thermodynamics, we have discussed its performability in the framework of black hole thermodynamics. The violation of the Bekenstein-Hawking law has been suggested as a viable solution for this problem. Moreover, we have shown that, by requiring that the entropy of extremal black holes is zero, one can support (U) from a ther-

${ }^{\mathrm{n}}$ For the value of the constant see also 31 
mostatic point of view. We remark that finding e.g. $S=0$ for extremal states does not mean that $(\mathrm{N})$ is valid, unless such a result is corroborated by a limit approach as $T \rightarrow 0^{+}$. In fact $(\mathrm{N})$ gets, as a matter of facts, its real meaning and its real experimental verifications in standard thermodynamics if it is intended not as the behavior of the entropy at exactly $T=0$, but as the limit of entropy differences as $T \rightarrow 0^{+}$.

The result of 4 (the appropriate frame is a dynamic one and an irreversible thermodynamic one) has been interpreted as a strong corroboration for the picture in which extremal states are separated by a discontinuity with respect to non-extremal ones. Qualitative arguments allowing to match Israel's theorem both with the hypothesis $S_{E}=0$ and with the interpretation of black hole entropy as entanglement entropy have also been introduced.

\section{Acknowledgments}

FB wishes to thank C.Destri and A.Parola for interesting discussions at the preliminary stages of this work. Thanks are also addressed by FB to S.Liberati for his interesting comments on a old version of this paper.

\section{Appendix A. Adiabatic transformations in black hole thermodynamics}

We determine the equations for reversible adiabatic transformations in black hole thermodynamics. In the non-extremal cases they correspond to isoareal transformations because of the Bekenstein-Hawking law.

Adiabatic transformations for non-extremal black holes satisfy the equation

$$
A=A_{0}=\text { const. }
$$

that in the general case of a Kerr-Newman black hole becomes

$$
r_{+}^{2}+\frac{J^{2}}{M^{2}}=C
$$

where $C=A_{0} /(4 \pi)$ is a positive constant. If one defines $x \equiv M^{2} ; y \equiv Q^{2} ; z \equiv J^{2}$ then the above equation is equivalent to the following one:

$$
2 x-y+2 \sqrt{x} \sqrt{x-\frac{z}{x}-y}=C .
$$

One can solve e.g. for $z$ and find

$$
z=C x-\frac{1}{4}(y+C)^{2} .
$$

It is easy to show that $x \in\left(x_{L}^{2} / C, x_{L}\right)$, where $x_{L} \equiv(y+C) / 2$. The extremal sub-manifold is defined by

$$
z_{E}=x^{2}-x y
$$


and its intersection with the above isoareal surface takes place at

$$
x-\frac{1}{2}(y+C)=0 \Leftrightarrow x=x_{L} .
$$

The extremal state surface and the isoareal surface are tangent. In fact, their tangent planes coincide along A.1.

We can conclude that there are two classes of adiabatic transformations: 1) standard adiabatic transformations for non-extremal black holes; they are isoareal and isentropic; 2) "extremal" adiabatic transformations that in general are not isoareal. In the Kerr-Newman extremal case it is possible to allow also for isoareal extremal transformations that can be obtained by imposing $d A_{E}=0$. Their equation is

$$
Q^{4}+4 J^{2}=\left(\frac{A_{0}}{4 \pi}\right)^{2}
$$

They represent a nontrivial sub-manifold of the general extremal case. If $J=0$ or $Q=0$ one gets that this manifold becomes a single point.

\section{Appendix B. Glassy systems, frozen equilibrium and (N)}

It is well known that a wide discussion about the validity of $(\mathrm{N})$ was given rise by some physical systems that seemed to violate Nernst's postulate of isentropy of the zero-temperature states. Actually, it has been shown that these peculiar systems (e.g. CO and glassy substances) don't satisfy the condition of internal equilibrium, that is, near $T=0$ some degrees of freedom remain frozen in a non-equilibrium meta-stable configuration 112457. Elements of configurational disorder can remain unchanged during the cooling down of the system towards a low temperature. The relaxation time to a condition of inner equilibrium is much bigger than the measurement time and it can be effectively infinite. A residual molar entropy is then allowed at $T=0$. Long-time measurements have shown the convergence to (N) of the calorimetric entropy for some substances violating $(\mathrm{N})$. Thus, a further hypothesis has been added in order to ensure the validity of $(\mathrm{N})$, which is the condition of internal equilibrium 1157. There is no definitive agreement about this hypothesis.

In the black hole case, it is still difficult to find out a definitive notion of "internal states" and of micro-states. "Meta-stability of non-equilibrium states" and "frozen-in disorder" can hardly justify the violation of $(\mathrm{N})$ in the case of black holes. The difference with respect to the case of the apparent violation of $(\mathrm{N})$ in "glassy systems" having finite relaxation times is evident, indeed by increasing the measurement time no convergence to the implementation of $(\mathrm{N})$ can be expected for black holes.

\section{Appendix C. Failure of concavity and $(\mathrm{U}) \Leftrightarrow(\mathbf{N})$}

In the following, we analyze what happens if condition a) is relaxed. In fact, in black hole thermodynamic a) is not satisfied. For simplicity of notation, we substitute $S_{T, x}$ 
for $S\left(T, x^{1}, \ldots, x^{n}\right)$ and $C_{x}$ for $C_{x^{1}, \ldots, x^{n}}$.

\section{Appendix C.1. Relaxing condition a) against $(U) \Rightarrow(N)$}

The presence of heat capacities with opposite sign can invalidate the proof of the double implication $(\mathrm{U}) \Leftrightarrow(\mathrm{N})$. Let us assume then that $(\mathrm{U})$ is satisfied and that there exist isometric curves (i.e. isometric transformations) reaching $T=0$ such that some have $C_{x}>0$ and other $C_{y}<0$. Note that the presence of heat capacities $C_{y}<0$ at constant deformation parameters means the failure of the standard concavity properties of the entropy. Then, this non-uniformity of the sign of the heat capacities along transformation reaching the absolute zero allows to violate the implication $(\mathrm{U}) \Rightarrow(\mathrm{N})$, and, moreover, it seriously jeopardizes the identification of (U) with the absence of isentropic reaching $T=0$. See fig. 6 Let us first consider the case where along different isometric transformations with opposite signs of the corresponding heat capacities and starting from $T=0$ it is possible to reach the same isentropic surface. Let us define

$$
\begin{aligned}
& S_{T_{1}, x}=S_{0, x}+\int_{0}^{T_{1}} \frac{C_{x}}{T} d T>S_{0, x} \\
& S_{T_{2}, y}=S_{0, y}-\int_{0}^{T_{2}} \frac{\left|C_{y}\right|}{T} d T<S_{0, y} .
\end{aligned}
$$

If $S_{T_{1}, x}=S_{T_{2}, y}$ and if $T_{1}, T_{2}>0$, the equality

$$
S_{0}+\int_{0}^{T_{1}} \frac{C_{x}}{T} d T=S_{0}-\int_{0}^{T_{2}} \frac{\left|C_{y}\right|}{T} d T
$$

is obviously impossible. The only possibility is that $T_{1}=0=T_{2}$, but, then, (U) cannot be implemented in general as "absence of isentropic transformation reaching $T=0$ ", except for a $S-T$ diagram of the type sketched in fig. 7

Note that this reasoning concerning the non-uniformity of signs of heat capacities for transformations connected to $T=0$ can be easily extended to the case where generic curves $\gamma^{0}$ which arrive at $T=0$ and having $C_{\gamma^{0}}<0$ are allowed.
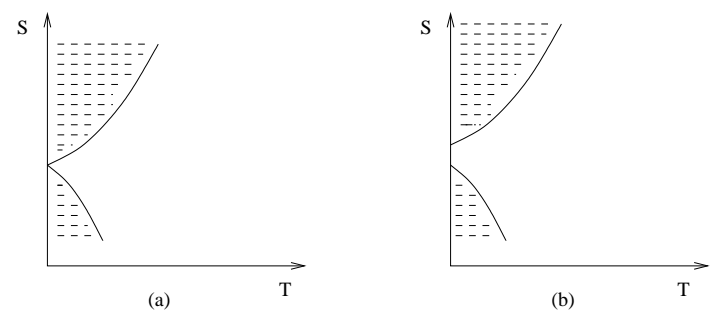

Fig. 6. Isentropes approaching $T=0$ at finite parameters exist and $(\mathrm{U})$ is ensured by a discontinuity in the sense of Landsberg. $(\mathrm{N})$ is allowed only in case (a).

If, instead, no intersection to the same isentrope is possible, then, again a violation of $(\mathrm{N})$ can occur. In the following figures, some possible diagrams are sketched. 
They imply a violation of Landsberg's hypothesis b) and/or of c).

As we have shown, $(\mathrm{N})$ can be allowed for only if the starting isentropic coincides with the zero-temperature one (cf. fig. below), but, if $S \geq 0$ is assumed, then at least Planck's restatement of $(\mathrm{N})$ has to be violated.

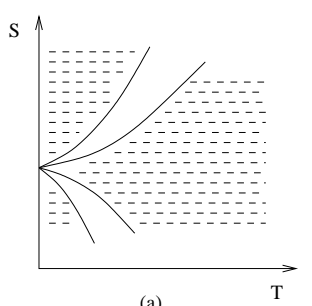

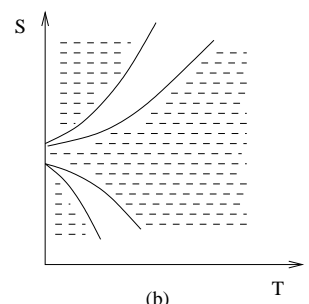

(b)

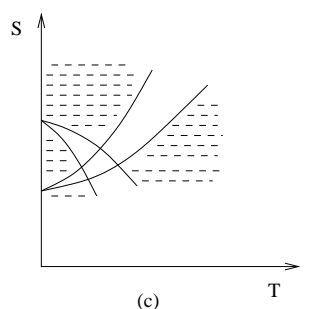

Fig. 7. A multi-branches structure is allowed. In all cases, (U) is ensured without invoking discontinuities near the absolute zero. In (a), in spite of the presence of $C_{y}<0$ connected to $T=0$, (N) holds. In (b) no isentrope is intersected both by curves reaching $T=0$ and having $C_{x}>0$ and by curves reaching $T=0$ with $C_{y}<0$. In (c), such an isentrope exists but no isentrope reaching the absolute zero is allowed. In (b) and in (c) the entropic version (N) is violated.

\section{Appendix C.2. Relaxing condition a) against $(N) \Rightarrow(U)$}

It is also easy to deduce that the existence of paths with opposite sign near $T=0$ could be reconciled with $(\mathrm{N})$ without implying (U). In fact, if $C_{x}>0, C_{y}<0$ then

$$
\begin{gathered}
S_{0}+\int_{0}^{T_{1}} \frac{C_{x}}{T} d T>S_{0} \\
S_{0}-\int_{0}^{T_{2}} \frac{\left|C_{y}\right|}{T} d T<S_{0}
\end{gathered}
$$

and, in absence of suitable multi-branching (hypothesis b)), the isentropic $S=S_{0}$

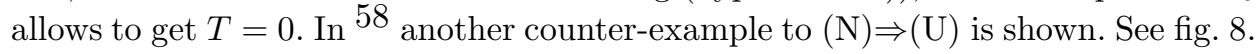
The system studied therein displays a particular behavior, in the sense that $S=0$ is attained at $T_{1}>0$ and $C_{p}=0$ for $0 \leq T \leq T_{1}$ is allowed. Condition a) is then violated. According to standard proofs, $S=S_{0}$ cannot be attained at $T>0$.

\section{Appendix D. Stability properties and (U)}

The entropy is a non-concave function even in the case in which the thermal stability is ensured (as near the extremal states). In fact, there are principal minors of the Hessian (see e.g. ${ }^{5960}$ ) that don't satisfy the concavity (stability) requirement. In 27 it is shown that in the black hole case the minor $\Delta_{3}$ does not implement the stability requirement for any value of the physical parameters. In fact 27

$$
\Delta_{3}=\frac{\pi}{8} \frac{1+3 \Omega^{2} Q^{2}-\Phi^{2}}{M T^{5} S^{3}}
$$



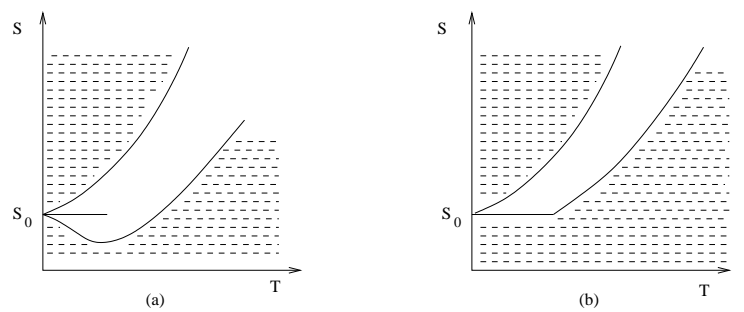

Fig. 8. Two examples of violation for the implication $(\mathrm{N}) \Rightarrow(\mathrm{U})$. (a) Paths having $C<0$ and reaching $T=0$ are allowed; (b) A behavior like the one of Wheeler's counter-example [58] is displayed (the original counter-example requires $S_{0}=0$ ).

and the stability requirement $\Delta_{3}<0$ is never satisfied. Moreover, the instability becomes maximal at the extremal limit (where all the minors diverge). We show explicitly this property in the Reissner-Nordström and in the Kerr cases. Let us define

$$
a_{X_{i} X_{j}} \equiv \frac{\partial^{2} S}{\partial X_{i} \partial X_{j}}
$$

where $X_{i}$ are the extensive variables appearing in the fundamental relation in the entropy representation. In the Reissner-Nordström and in the Kerr cases we get respectively

$$
\begin{aligned}
a_{M M}^{\mathrm{RN}} & =-(2 \pi) \frac{1}{\left(M^{2}-Q^{2}\right)^{3 / 2}}\left(M+\sqrt{M^{2}-Q^{2}}\right)^{2}\left(M-2 \sqrt{M^{2}-Q^{2}}\right) \\
a_{Q Q}^{\mathrm{R} N} & =-(2 \pi) \frac{1}{\left(M^{2}-Q^{2}\right)^{3 / 2}}\left(M^{3}+\left(M^{2}-Q^{2}\right)^{3 / 2}\right) \\
a_{M Q}^{\mathrm{RN}} & =(2 \pi)^{2} \frac{Q^{3}}{\left(M^{2}-Q^{2}\right)^{3 / 2}} \\
a_{M M}^{\mathrm{Kerr}} & =(4 \pi) \frac{1}{\left(M^{4}-J^{2}\right)^{3 / 2}}\left(M^{6}-3 M^{2} J^{2}+\left(M^{4}-J^{2}\right)^{3 / 2}\right) \\
a_{J J}^{\mathrm{Kerr}} & =-(2 \pi) \frac{M^{4}}{\left(M^{4}-J^{2}\right)^{3 / 2}} \\
a_{M J}^{\mathrm{Kerr}} & =(4 \pi) \frac{J M^{3}}{\left(M^{4}-J^{2}\right)^{3 / 2}}
\end{aligned}
$$

and

$$
\begin{aligned}
\left(a_{M Q}^{\mathrm{R} N}\right)^{2}-a_{M M}^{\mathrm{R} N} a_{Q Q}^{\mathrm{R} N}= & (2 \pi)^{2}\left(M^{2}-Q^{2}\right)^{-3 / 2} \\
& {\left[M^{3}+\left(4 M^{2}-Q^{2}\right) \sqrt{M^{2}-Q^{2}}+3 M^{2}\left(M^{2}-Q^{2}\right)\right] } \\
\left(a_{M J}^{\mathrm{Kerr}}\right)^{2}-a_{M M}^{\mathrm{Kerr}} a_{J J}^{\mathrm{Kerr}}= & 8 \pi^{2} M^{4}\left(M^{4}-J^{2}\right)^{-2}\left(M^{2}+\sqrt{M^{4}-J^{2}}\right) .
\end{aligned}
$$

In the case of two independent thermodynamic variables $X_{1}, X_{2}$, stability requires that $a_{X_{1} X_{1}} \leq 0, a_{X_{2} X_{2}} \leq 0,\left(a_{X_{1} X_{2}}\right)^{2}-a_{X_{1} X_{1}} a_{X_{2} X_{2}} \leq 0 \underline{60}$. The third condition in both cases is always violated and moreover a divergence in the extremal limit appears. The condition that the entropy hypersurface lie everywhere below its family 
of tangent hyperplanes $\underline{60}$ is so violated maximally near the extremal states. Such an instability can give a thermodynamic reason for a thermodynamic "runaway" from extremal states and, as a consequence, also for (U).

\section{References}

1. B.Bardeen, B.Carter and S.W.Hawking, Commun. Math. Phys. 31, 161 (1973).

2. B.Bardeen, Nature 226, 64 (1970).

3. P.C.W.Davies, Proc. R. Soc. Lond. A353, 499 (1977).

4. W.Israel, Phys. Rev. Lett. 57, 397 (1986). W.Israel, in Black Hole Physics, eds. V. de Sabbata and Z.Zhang (Kluwer Academic, Dordrecht, 1992), p. 150.

5. R.Wald, Phys. Rev. D56, 6467 (1997).

6. I.Racz, Class. Quant. Grav. 17, 4353 (2000).

7. S.W.Hawking, G.T.Horowitz and S.F.Ross, Phys. Rev. D51, 4302 (1995).

8. S.W.Hawking and G.T.Horowitz, Class. Quantum Grav. 13, 1487 (1996).

9. L.Vanzo, Phys. Rev. D55, 2192 (1997).

10. T.Brotz and C.Kiefer, Phys. Rev. D55, 2186 (1997).

11. F.E.Simon, Z. Naturforschg. 6a, 397 (1951)

12. M.W.Zemansky and R.H.Dittman, Heat and Thermodynamics, An Intermediate Textbook (McGraw-Hill, New York, 1981).

13. P.T.Landsberg, Thermodynamics with quantum statistical illustrations (Interscience Publishers, New York, 1961).

14. P.T.Landsberg, Rev. Mod. Phys. 28, 363 (1956).

15. P.T.Landsberg, Thermodynamics and Statistical Mechanics (Dover, New York, 1990).

16. A.Münster, Statistical Thermodynamics. Vol. 1 (Springer-Verlag, Berlin, 1969).

17. J.Preskill, P.Schwarz, A.Shapere, S.Trivedi and F. Wilczek, Mod. Phys. Lett. A6, 2353 (1991).

18. Th.M.Nieuwenhuizen, Phys. Rev. Lett. 81, 2201 (1998).

19. F.Belgiorno, J. Phys. A: Math. Gen. 36, 8165 (2003).

20. F.Belgiorno, J. Phys. A: Math. Gen. 36, 8195 (2003).

21. H.A.Buchdahl, The Concepts of Classical Thermodynamics (Cambridge University Press, Cambridge 1966).

22. R. Haase, in Physical Chemistry. An Advanced Treatise, eds H.Eyring, D.Henderson and W.Jost (Academic, New York, 1971). Vol. I, p.1.

23. A.Münster, Statistical Thermodynamics. Vol. 2 (Academic Press, New York, 1974).

24. E.A.Guggenheim, Thermodynamics. An advanced treatment for chemists and physics (North-Holland, Amsterdam, 1949).

25. P.T.Landsberg, in Black Hole Physics, eds. V. de Sabbata and Z.Zhang (Kluwer Academic, Dordrecht, 1992), p. 99.

26. E.A.Martinez, Phys. Rev. D54, 6302 (1996).

27. D.Tranah and P.T.Landsberg, Collective Phenomena 3, 81 (1980).

28. C.O.Lousto, Nucl. Phys. B410, 155 (1993); Erratum-ibid. B449, 433 (1995).

29. L.Smarr, Phys. Rev. Lett. 30, 71 (1973). Erratum-ibid., 30, 521 (1973).

30. F.Belgiorno, Phys. Lett. A312, 324 (2003).

31. F.Belgiorno, J. Math. Phys. 44, 1089 (2003).

32. F.Belgiorno, Homogeneity as a Bridge between Gibbs and Carathéodory. math-ph/0210011 (2002).

33. A.B.Pippard, Elements of Classical Thermodynamics (Cambridge University Press, Cambridge, 1957). 
October 23, 2018 13:9 WSPC/Guidelines bhthird1

34. The Collected Papers of Albert Einstein. 4, The Swiss years: writings, 1912-1914. Eds. A.J.Kox and R.Schulmann (Princeton University Press, Princeton, 1996). p. 266-270.

35. A.Ghosh and P.Mitra, Phys. Rev. Lett. 78, 1858 (1997).

36. C.Kiefer and J.Louko, Ann. Phys. (Leipzig) 8, 67 (1999).

37. J.D.Bekenstein, Black Holes: Classical Properties, Thermodynamics, and Heuristic Quantization. Preprint, gr-qc/9808028 (1998).

38. N.Dahdich, K.Narayan, Phys. Lett. A231, 335 (1997).

39. G.W.Gibbons and R.E.Kallosh, Phys. Rev. D51, 2839 (1995).

40. S.Liberati and G.Pollifrone, Phys. Rev. D56, 6458 (1997).

41. Bin Wang and Ru-Keng Su, Phys. Lett. B432, 69 (1998).

42. B.Carter, in Black Holes. Proceedings of the summer school, Les Houches. Eds. C. DeWitt and B.S.DeWitt (Gordon and Breach, New York, 1973), p. 57.

43. D.W.Sciama, Vistas in Astronomy 19, 385 (1976).

44. T.A.Roman, Gen. Rel. Grav. 20, 359 (1988).

45. Ch.J.Farrugia and P.Hajicek, Commun. Math. Phys. 68, 291 (1979).

46. M.Proszynski, Gen. Rel. Grav. 15, 403 (1983).

47. B.T.Sullivan and W.Israel, Phys. Lett. A79, 371 (1980).

48. M.K.Parikh and F.Wilczek, Phys. Rev. Lett. 85, 5042 (1999).

49. P.R.Anderson, W.A.Hiscock and B.E.Taylor, Phys. Rev. Lett. 85, 2438 (2000).

50. S.Liberati, T.Rothman and S.Sonego, Phys. Rev. D62, 024005 (2000); Int. J. Mod. Phys. D10, 33 (2001).

51. S.Hod, Phys. Rev. D61, 084018 (2000).

52. B.Carter, in General Relativity: an Einstein Centenary Survey, eds. S.W.Hawking and W.Israel (Cambridge University Press, Cambridge, 1979), p. 294.

53. T.Damour, in Proceedings of the second Marcel Grossmann Meeting on General Relativity, ed. R. Ruffini (North-Holland, Amsterdam, 1982), p. 587.

54. D.W.Sciama, in Centenario di Einstein (Editrice Giunti Barbera-Universitaria, Firenze 1979), p. 617.

55. D.W.Sciama, P.Candelas, D.Deutsch, Adv. Phys. 30, 327 (1981).

56. L.Bombelli, R.K.Koul, J.Lee, R.Sorkin, Phys. Rev. D34, 373 (1986).

57. A.H.Wilson, Thermodynamics and statistical mechanics. (Cambridge University Press, Cambridge, 1957);

J.Wilks, The Third Law of Thermodynamics (Oxford University Press, London, 1961).

58. J.C.Wheeler, Phys. Rev. A43, 5289 (1991).

59. P.T.Landsberg and D.Tranah, Collective Phenomena 3, 73 (1980).

60. H.B.Callen, Thermodynamics and an introduction to Thermostatistics (Wiley, New York, 1985). 